\title{
Enhancing Social based Routing Approach using Grey Wolf Optimization in Vehicular ADHOC Networks
}

\author{
Sarvada Sharma \\ M.Tech Research Scholar \\ Department of CSE \\ Amritsar College of Engineering and Technology
}

\author{
Sandeep Kad \\ Associate Professor \\ Department of CSE \\ Amritsar College of Engineering and Technology
}

\begin{abstract}
VANET (Vehicular ad-hoc network) is a formation of intelligent vehicles with the plan to defeat the transportation issue and consequently diminishes the accident proportion. Since VANET is in like way a sort of MANET enclosed by human-driven center points, the social idea can be utilized to comprehend the routing decisions in VANET. This propels investigators to procure the possibility of Social Network Analysis (SNA) to develop routing plans. The serious issue in any system is the drop of parcel. Social characteristics based framework similarly face such issues which results in wasteful message conveyance. In this paper, grey wolf optimization is applied on social based routing scheme for fixed line VANET with the end goal to diminish the drop of parcel and enhance the throughput of the network. This is made possible with system centrality analysis by shapely value which enhances the choice by wolf optimization approach. Other than this, comparison is done between the social based fixed line routing scheme and the proposed work with GWO. The conduct of the utilizing nodes availability by its centrality calculation will then be observed. The parameters utilized in this paper to quantify the viability of the optimized routing scheme are Throughput, Delay, PDR, Latency, Buffer Time and Hop count.
\end{abstract}

\section{General Terms}

Network, community, grey wolf optimizer, VANET, MANET, social characteristics, vehicles, nodes, hubs, packets, message.

\section{Keywords}

VANET, MANET, GWO, Community, IKC, BFD, ECFD, ICFD.

\section{INTRODUCTION}

\subsection{VANETs}

Vehicular systems have picked up an expanding research enthusiasm for the last late years, because of their wide scope of potential application situations including, yet not restricted to, systems to scatter safety related data [25]. A VANET is a remote system that is framed between vehicles on an asrequired premise. Every vehicle's remote system range might be restricted to a couple of hundred meters, so giving start to finish correspondence over a bigger separation expects messages to jump through a few hubs. Infrastructure isn't required for a VANET to be made, albeit changeless system hubs might be utilized as roadside units. These roadside units open up a wide assortment of administrations for vehicular systems, for example, going about as a slanted edge for messages on scantily populated streets, serving up geologically applicable information, or filling in as a portal to the Internet [1]. A standout amongst the most significant angles that decide the achievement of VANET is the solid message routing from a source hub to a goal hub. Directing in VANET depends on the nearness of an adequate number of VANET hubs that establish strong ways to permit the sending of messages in the system. These ways can be influenced by the vehicles' versatility and traffic thickness, rapid topology changes making them unsustainable and questionable. In this manner, the structure of an effective routing protocol for VANET is viewed as a basic issue [2]. VANETs are an actualization of mobile ad hoc networks (MANETs). MANETs have no fixed framework and rather depend on customary hubs to perform directing of messages and system the network capacities. In this way, various research provokes should be tended to for between vehicular correspondences to be broadly conveyed. For instance, directing in traditional mobile ad hoc networks is a difficult errand in view of the system's dynamic topology changes. Various investigations and proposition of routing protocols have been led to transfer information in such a unique situation; anyway these arrangements can't be connected to the vehicular condition because of the particular requirements and qualities of VANETs [11]. Figure 1 represents the vehicular social networks and figure 2 represents the architecture of VANETs.

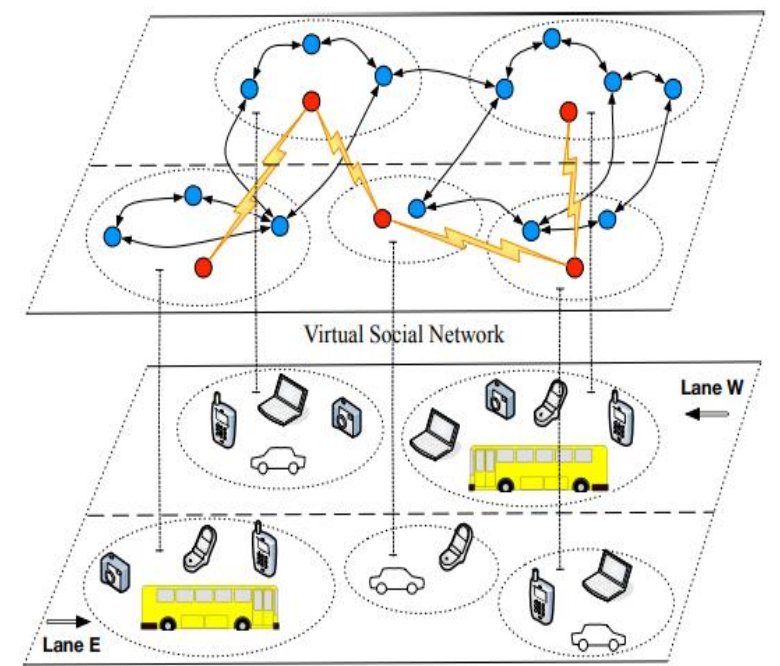

Fig 1: Vehicular Social Networks 


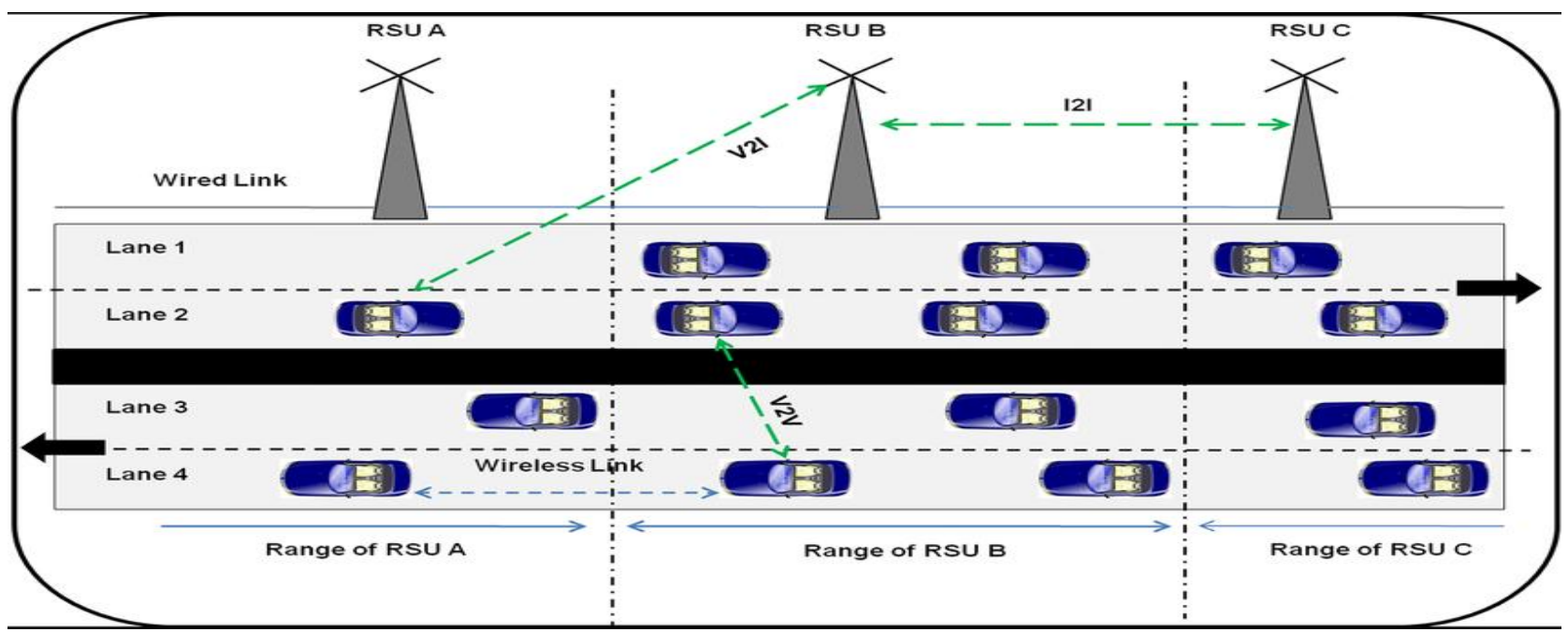

Fig 2: Architecture of VANET [11]

\subsection{Social-Based Routing Scheme}

As of late, the social-based systems have been worked to bring various gatherings of individuals inside range for potential correspondence. Such social-based systems are not just used to associate the PCs for worldwide interchanges arrange however it can likewise be utilized to interface vehicles in urban conditions. Social-based steering in Vehicular Ad hoc Networks (VANET) is pulled in the consideration of research network where the traffic data enables us to settle on better directing choices. VANET gives the capacity to vehicles to impart remotely among close-by vehicles and street side remote sensors to exchange data for safe driving, powerful route arranging, versatile detecting and in-vehicle excitement [12]. It is trusted that hubs in same network have more chances to contact and are probably going to meet consistently. Inside the network, a few people are progressively prevalent, and interface with a greater number of individuals than others [17].

\subsubsection{Benefits}

1. The improvement of routing adequacy in VANET can be made possible by perceiving the social conduct of individuals and this become very simple amid going as individuals with unlike nature and economic status comes in contact.

2. By distinguishing group's social qualities (say tie quality), the hub can redesign routing by carrying the message to the frequently experienced vehicles as opposed to delivering traffic in the system by sending the message in deception.

3. Social connection is an unmistakable factor influencing the most appropriate plan of routing for VANET with respect to a large portion of the general population. This dormant trademark results in unsurprising steering data and lessening in number of overhead [24].

\subsection{Grey Wolf Optimization}

This algorithm's fundamental idea is reproducing the conduct of grey wolf living in a pack. They have genuine chain of importance of social strength. Alpha is known as the dimension heads and is in charge of basic leadership in the pack. Beta is known as the second dimension subordinate wolves that help in settling on choice for alpha or different exercises. Delta is known as the third dimension subordinate wolves. This classification part comprises of elders, scouts, hunters, caretakers and sentinels. For locale limit perception and in any risk case, scouts are at risk for notice. The assurance and pack's security ensure is given by sentinels. The ability wolves are the older folks. Alphas and betas are helped by seekers while prey chasing and thinking about the evil, feeble, and injured wolves via guardians and giving nourishment to pack. Omega is the most minimal dimension. Grey wolves have the capacity of remembering the prey position and surrounding them. The alpha as a pioneer performs in the chase. For reenacting the grey wolves chasing conduct in the numerical model, accepting the alpha $(\alpha)$ is the best arrangement. The second ideal arrangement is beta $(\beta)$ and the third ideal arrangement is delta $(\delta)$. Omega $(\omega)$ is thought to be the applicant arrangements. Alpha, beta and delta directs the chasing while position ought to be refreshed by the omega wolves by these three best arrangements considerations [23].

Rest of the paper is organized as follows. Section II includes the literature review regarding all the work done in the research of VANET that includes the social behavior of the vehicles involved in the network. Section III presents the proposed work including the grey wolf algorithm and a flowchart of how it works. Section IV shows the results of the proposed work and its comparison with the existing work to prove that after optimizing the base work with GWO the results are enhanced to much extent. Section V represents the conclusion of the paper and the future scope of the proposed work. Section VI is for the references.

\section{LITERATURE REVIEW}

To efficiently propagate social phenomenon in VANETs, literature survey explores all the researches related to social based routing algorithms in vehicular networks. In [12], a fuzzy-assisted social-based routing (FAST) convention is presented. FAST utilizes people's social conduct to optimize the routing choices. So as to exchange information from the place of origin to goal, past data related to traffic is utilized. According to the reenactments, FAST outcomes in 32\% high conveyance proportion, $80 \%$ low delays and lower the hop counts to half.

In [6], so as to settle on routing choices, understanding of energy is utilized as the fundamental element. The utilization of social attributes among hubs prompts the exhaustion of energy assets and the reason was that the hubs are 
impermanent and there are almost none appropriate routing choices. The methodology utilized in this paper upgrades the delivery procedure just as the energy utilization between the hubs is additionally adjusted.

Li-Der Chou et.al. [7] this paper proposed a convergence based routing convention based on the course of bundle trade and the vehicle's heading direction. IBR fortifies particular development conditions at the combinations, and precise estimations of bundle controlling postponements were in addition proposed. In expansion, a system mapping the parameters of the Manhattan structure model to the onedimensional street condition was proposed.

In [9], a social contribution-based routing protocol (SCR) is developed. The fundamental contemplation of this protocol is the likelihood of conveying the packet to the goal and the amount of social contribution a hub make to advance the message. These two elements help in making the selfish hubs progressively agreeable. On the off chance that the likelihood of high conveyance of message and the social commitment is low of any hub, at that point that hub is viewed as the most ideal next contender for delivery. The recreation results based on this thought demonstrate to be powerful and effective.

Wen-Hsing Kuo et.al. [13] have proposed a plan PBV2V that uses the pheromone thickness dissemination. By intermittently trading data with its neighbors and resuscitating its own table, the vehicle can locate the goal without broadcasting extra bundles over the framework. This declines the framework overheads and the pursuit time. The increase comes about to show that the PBV2V performs well the degree that the achievement rate and the ordinary skip check. Despite the vehicle thickness, the course of action's success rate can achieve $80 \%$ of the perfect game plan.

In [26], a social prominence based routing (SPBR) algorithm is proposed. It takes into account the contact time and multibounce neighbor information. As an issue of first significance a system is familiar which decisively perceive the idea of association between hubs. By then a social predominance is proposed to survey the social force of hub in the framework. SPBR settles on the coordinating decisions subject to the popularity, driving message closer to objectives with low routing hubs and network resources. Results show that the proposed computation basically upgrades the directing appeared differently in relation to Epidemic, Prophet and First Contact (FC), especially SPBR is achieve somewhere near $55.1 \%$ in overhead extent and higher by about $22.2 \%$ in conveyance rate than Epidemic when there are 40 hubs in the frameworks.

In [3], Eyuphan Bulut et.al. at first presented another estimation for isolating the possibility of associations unequivocally. Utilizing the presented estimation, every hub portrays its family relationship bundle as the strategy of hubs having association with itself either obviously or in a roundabout way. By at that point, it shows Friendship Based Directing in which quickly separated associations are utilized to settle on the sending choices of messages.

\section{PROPOSED WORK}

In VANET it should realize that information is being exchanged from which hub to which other hub. Its conduct ought to be refined for example it should realize what the parcel drop is or bundle exchange rate. There ought to be Nto-N refinement. For every one of these issues, it utilize Grey wolf optimization (GWO) that plays out a refinement for example layer by layer $(\alpha, \beta, \delta, \omega)$. It initially chooses the arrangement at that point refines the arrangement and finally characterizes the arrangement. In this hierarchy of optimization, which demonstrates social conduct and choice, will refine by various layers. In network's concern, routing decision is significant. On the off chance that it is refined layer by layer, at that point it will give compelling and effective outcomes for decreasing overhead and increment throughput. This segment clarifies the execution of the proposed work which is clarified in the given flowchart. This flowchart clarifies the well ordered working of the system by applying grey wolf optimization on it. Subtleties of this work are talked about beneath the flowchart by clarifying each progression in detail.

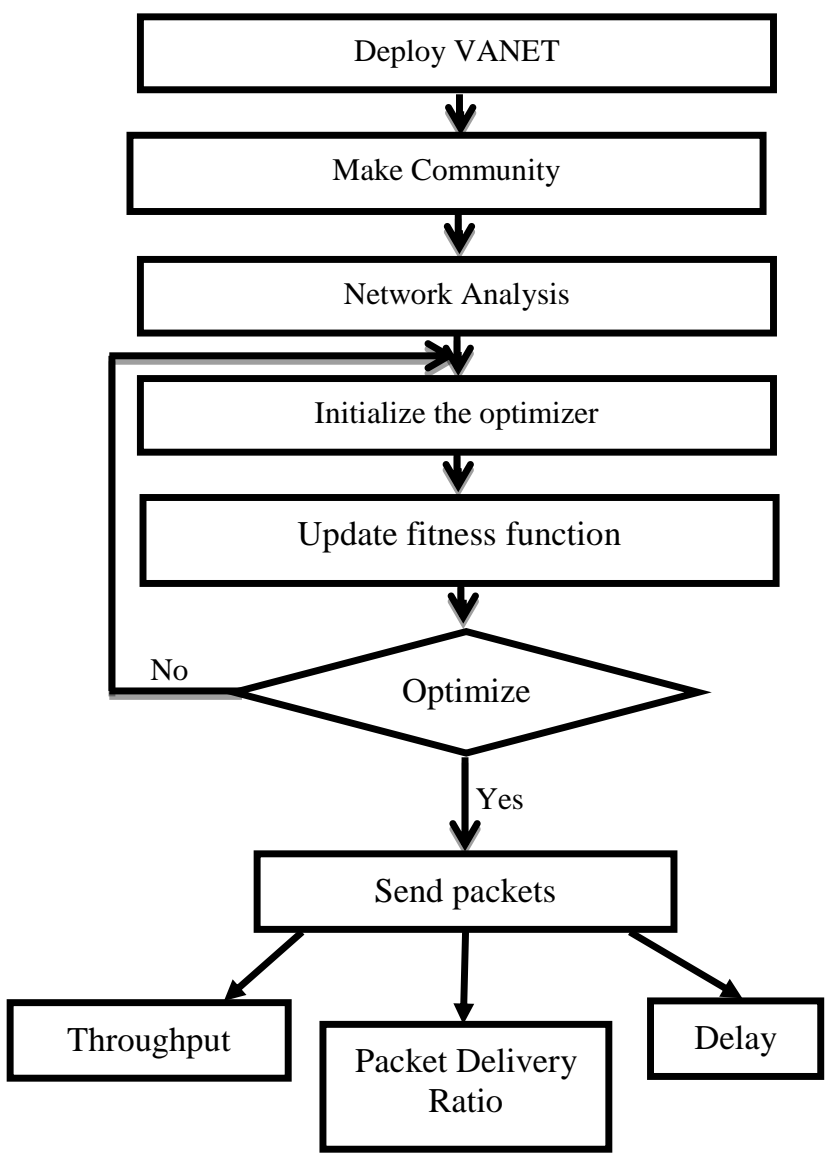

Fig 3: Flowchart for proposed work

Step1: The first and the foremost step of this flowchart is the formation of the VANET (Vehicle ad-hoc Network).

Step2. Next step is to make the community of the passenger nodes in a network. This community is made on the basis of the contact regularities of the nodes. Nodes with high contact regularities are put together in a same community. All this community work is done using an Improved k-clique Community detection algorithm (IKC).

Step3. Once the community is made, network analysis can be performed.

Step4. After the completion of the network analysis, instate the GREY WOLF optimizer.

Step5. GREY WOLF optimizer initiation will then updates the fitness of the solutions.

Step6. After this, check the system is streamlined or not, on the off chance that indeed, at that point exchanges the information parcel generally, otherwise again there will be a 
need to optimize the system.

Step7. In the event that the system is advanced and bundle is sent, at that point measure the three parameters throughput, delay, probability delivery ratio.

\subsection{Implementation Highlights}

\begin{tabular}{c} 
Algorithm \\
\hline I $=1$, Input VANET network \\
GWO Initialization \\
Initialize Wolves by subset of nodes. \\
Initialize a, A and C \\
Size of wolves=Number of subset of nodes \\
Estimate the $G_{\alpha}, G_{\beta}, G_{\delta}$ \\
iter $=1$ \\
repeat \\
for I to $G_{P}$ (Subset of nodes) \\
Update optimize location \\
End for \\
fitness value for $G_{\alpha}, G_{\beta}, G_{\delta}$ \\
update vector a, A, and C \\
iter $=$ iter+1 \\
Analyze the results of Routing results. \\
Routing is updated by $G_{\alpha}$. \\
Entil iter > maximum number of iteration \\
(stopping criteria) \\
output $G_{\alpha}$ \\
End
\end{tabular}

This algorithm clarifies how GWO helps in improving the social based directing plan. First of all the VANET formation is done by accepting the input as 1 . In the wake of conveying the system, GWO introduction is done so as to locate the fittest arrangement. For this, the wolves or the hubs in the system are introduced as the subset of hubs. Assume on the off chance that there are 10 hubs in the system, at that point there will be $2^{\wedge} 10$ subset of hubs and out of these subsets it need to locate the best subset. At that point the three primary parameters of GWO for example a, A and C are introduced where $\mathrm{A}$ and $\mathrm{C}$ are the coefficient vectors and a will be a parameter. These 3 are introduced by the objective function. After this it will set the span of the wolves which is equivalents to the quantity of subset of hubs determined by the grant procedure. The three best applicant arrangements are then instated i.e. $G \alpha, G \beta$ and $G \delta$. At first the iteration number is set to 1 and the procedure will rehash until the quantity of iterations is not exactly the most extreme number of iterations. The upgraded area of the hubs will continue refreshing for I to the subset of hubs. The fitness values $\mathrm{s}$ are then determined for $\mathrm{G} \alpha, \mathrm{G} \beta$ and $\mathrm{G} \delta$ again by utilizing the objective function. The three vectors $\mathrm{a}, \mathrm{A}$ and $\mathrm{C}$ are then refreshed. Each time the circle runs, the estimation of the cycle continues expanding by 1 and the procedure won't stop until it achieves its greatest number. Also, finally the system will get the output as G_a. Subsequently, through GWO the routing is refreshed by $\mathrm{G}_{-} \alpha$ and the outcomes are analyzed.

\section{RESULTS AND DISCUSSIONS}

In this section, the simulation results obtained after applying an optimization algorithm (GWO) are discussed in detail. The performance of the proposed VANET, which is developed in ONE simulator, is discussed below. The comparison of performance parameters is also discussed between the proposed and the existing work in order to determine the efficiency and effectiveness of the proposed work with GWO in VANET. Simulation parameters are shown in table 1.

Table 1: Simulation Parameters

\begin{tabular}{|c|c|}
\hline Simulation Time & $15000 \mathrm{sec}$ \\
\hline No.of wireless nodes & $100-500$ \\
\hline Speed & $50-100 \mathrm{KMpH}$ \\
\hline Routing algorithm & HibOp \\
\hline Simulated area & $1000 * 1000 \mathrm{~m}^{2}$ \\
\hline Packet size & 512 bytes \\
\hline Packet rate & Random-walk \\
\hline Mobility-model & Two-ray ground \\
\hline Propagation-model & \\
\hline
\end{tabular}




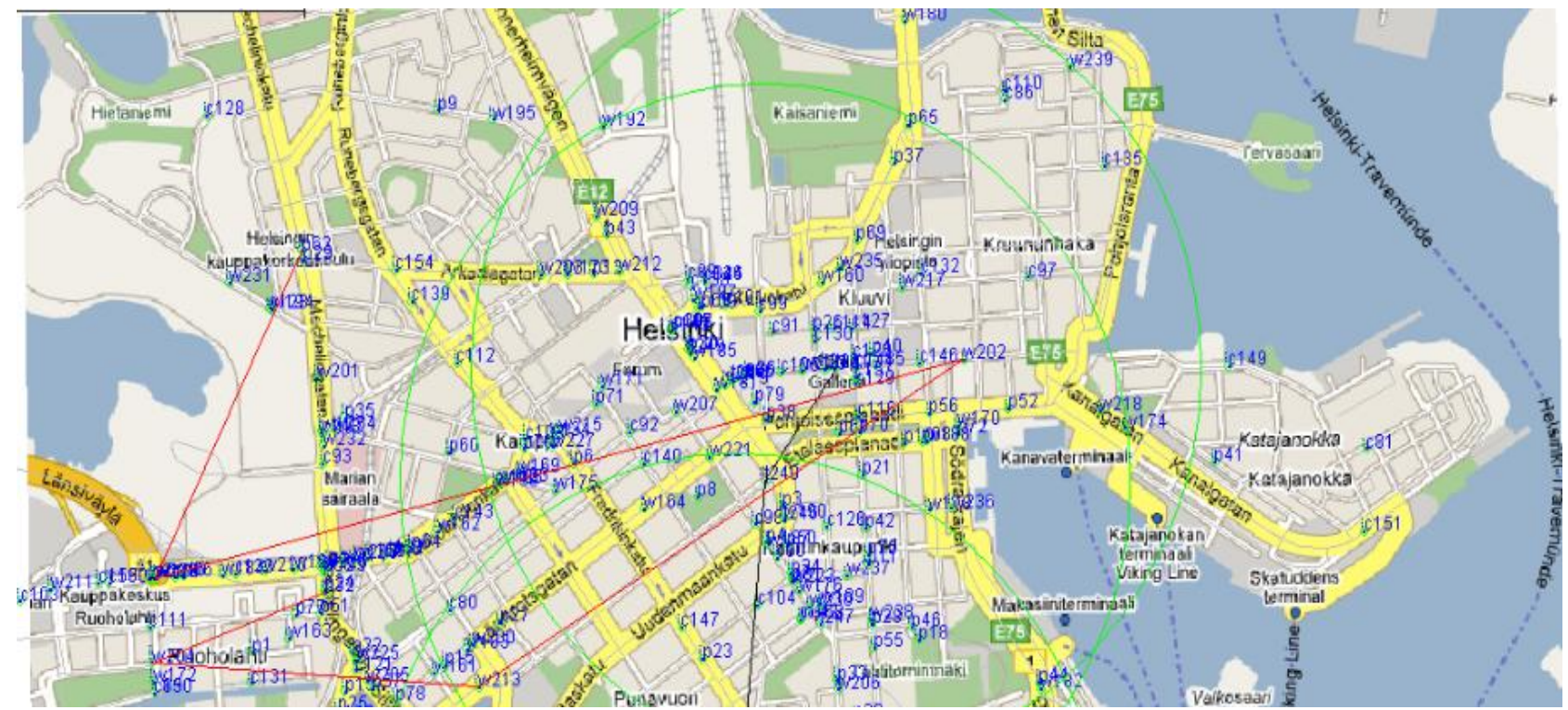

Fig 4: VANET Area

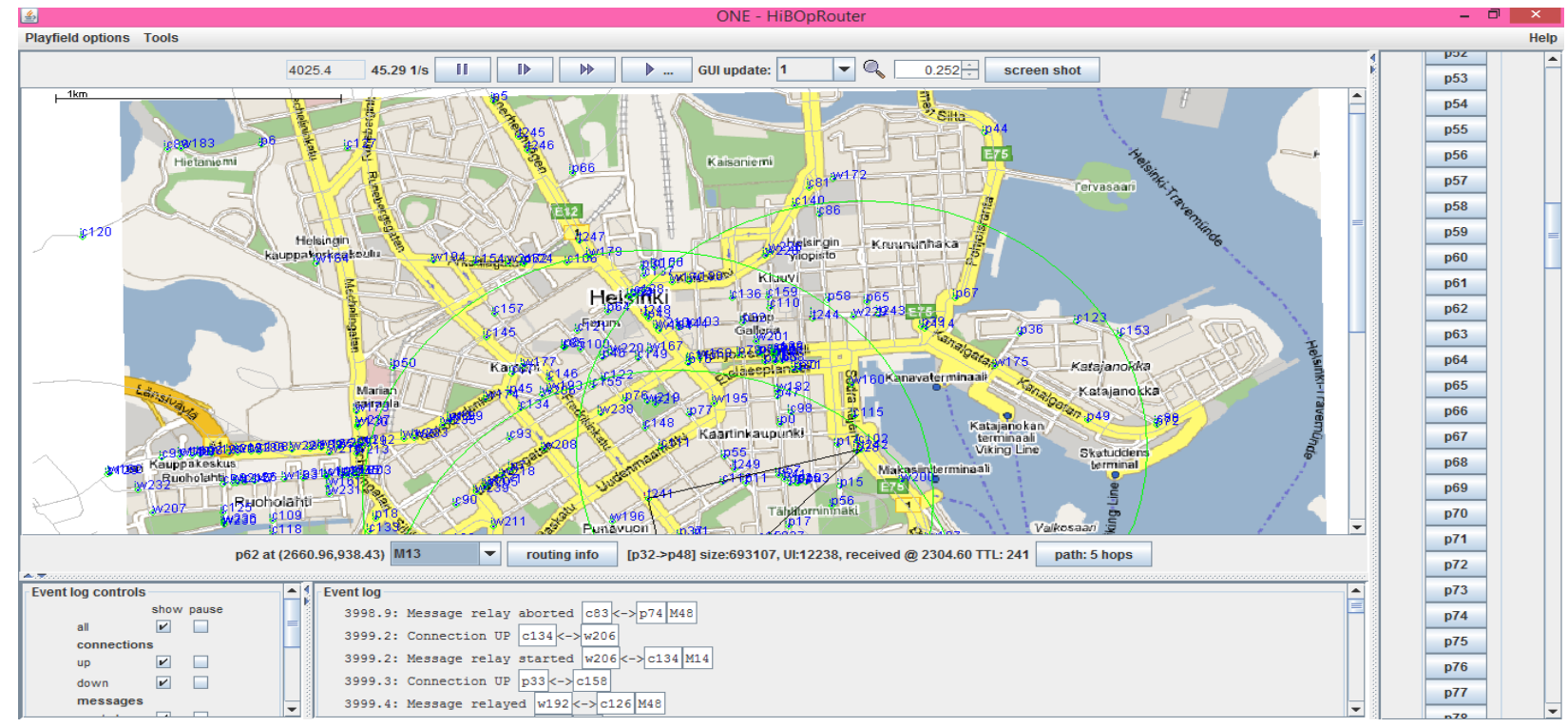

Fig 5: Simulation Screen

Figure 4 demonstrates the availability of hubs in dynamic VANET arrange. It indicates how the hubs are being associated with one another in a system. The huge green circles demonstrate a network or a gathering, wherein each taking an interest hub can speak with one another hub at any given moment. The red line in the figure speaks to the way of the bundle to be sent from source to goal. This red line additionally demonstrates the present association between two hubs. Heaps of such associations are being made and break during simulation. Figure 5 represents the full screen of simulation area of size $1000 * 1000 \mathrm{~m} 2$, in which the panel at the right side demonstrates every one of the nodes taking part in the system. By clicking on any of the nodes during the simulation, point by point data about that node will be appeared under the simulation area in an alternate panel like the position of that node, directing data of that hub. At the base of screen there is an event log which clarifies every one of the associations which are being built up and breakdown amid the simulation alongside the messages being dropped and conveyed. The presentation of VANET as far as throughput, latency, Probability Delivery Ratio, hop count and buffer time are talked about underneath.

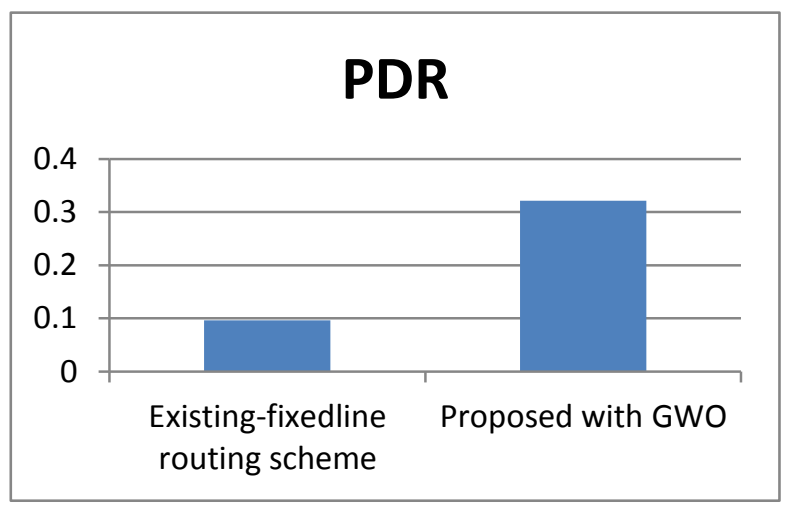

Fig 6: Probability Delivery Ratio 


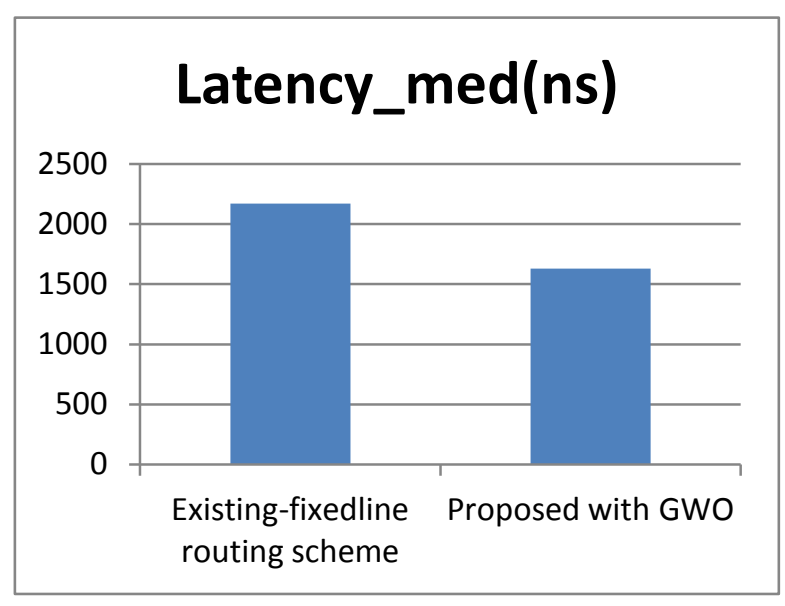

Fig 7: Latency (In Nano Seconds)

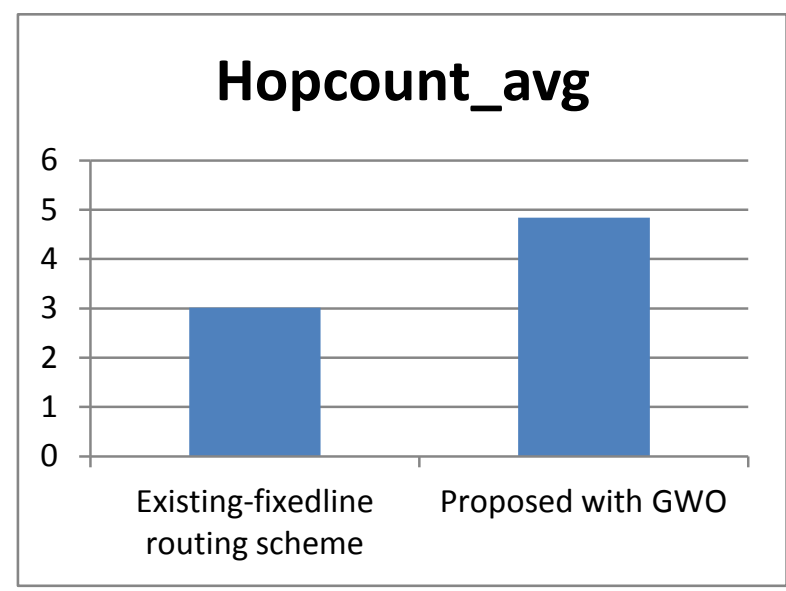

Fig 8: Hop Count Average

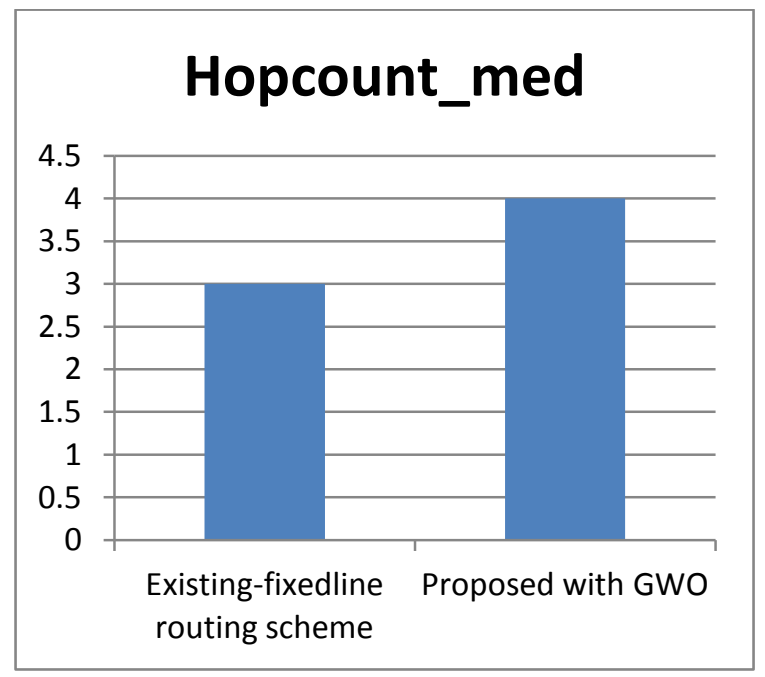

Fig 9: Hop Count Medium

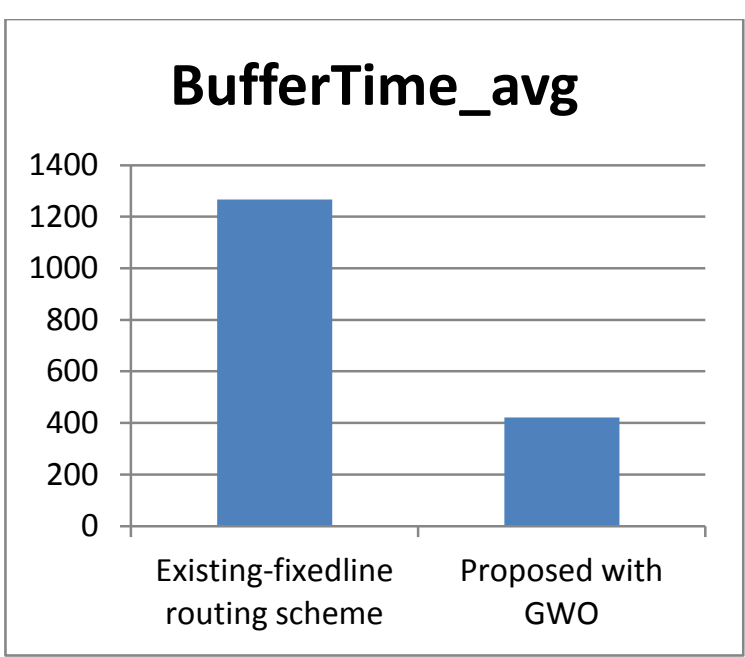

Fig 10: Buffer Time Average

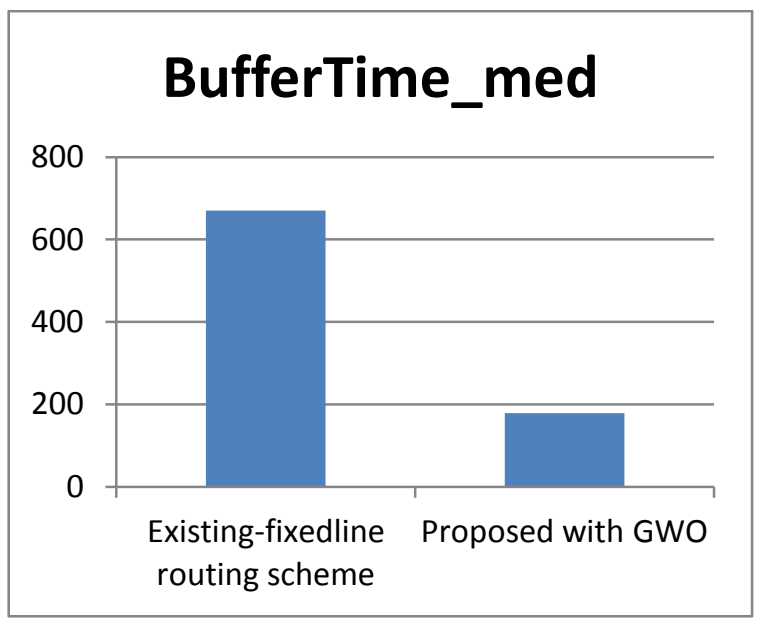

Fig 11: Buffer Time Medium

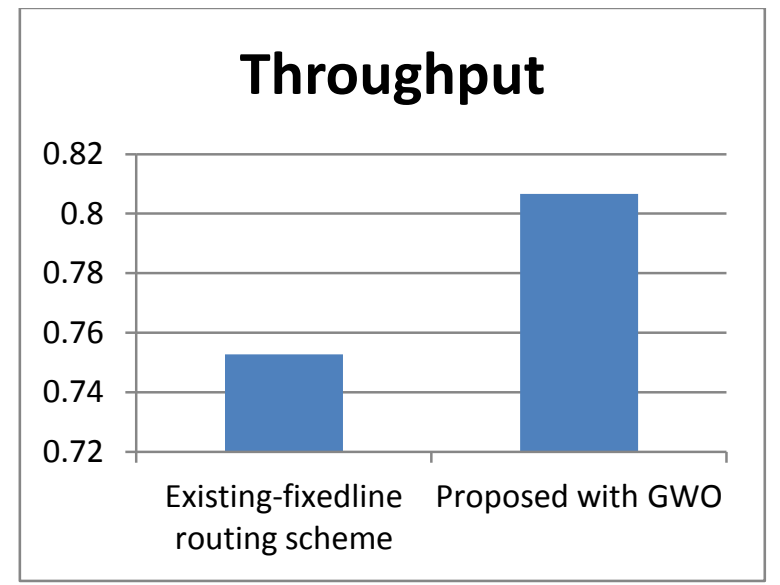

Fig 12: Throughput

The above graphs represent the parameters comparison between the existing-fixed line social based routing scheme and the same after applying the optimization technique (GWO) on it. Fig 6 indicate the graph plotted for first parameter i.e. PDR (Probability of delivery ratio). X-axis represents the values of the PDR and Y-axis shows the 2 bars for existing and the proposed work showing their values for PDR i.e. 0.0965114999 (existing value) and 0.320977165 (proposed value). It is clear from the graph that the PDR value increases in case of the proposed work as compared to the 
existing routing scheme. PDR is calculated as:

$$
\mathrm{PDR}=\frac{\text { total number of packets delivered successfully }}{\text { total number of packets sent }} \%
$$

Fig 7 represents the graph plotted for latency value. Latency is the term used to indicate any kind of delay in the transmission of data. X-axis represents the values for the latency and y-axis shows 2 bars indicating the values existing and the proposed work. After comparison the value of latency for the existing work comes out to be $2170.4 \mathrm{~ns}$ and for the proposed work it was $1628.869777 \mathrm{~ns}$. So it is very clear that optimization improves the latency to much extent. Fig 8 and Fig 9 shows the graph plotted for hop count average and hop count med. Hop count is the number of nodes a packet travels to in between its source and destination. X-axis of the graphs shows the values for the hop count and y-axis shows the values of hop count both for the existing and the proposed work. Hop count average for the existing work is 3.0145 and its 4.8357 for the proposed work. Hop count med value for the existing work is 3 and its 4 for the proposed work. Fig 10 and Fig 11 represents the graphs plotted for the buffer time average and buffer time med respectively. In case of buffer time average the values for the existing and the proposed work comes out to be $1266.0536 \mathrm{sec}$ and $421.0621 \mathrm{sec}$ respectively. In case of buffer time med the values comes out to be $669.8000 \mathrm{sec}$ for the existing work and $178.4000 \mathrm{sec}$ for the proposed work. The comparison itself explains the efficiency of the proposed GWO. Fig 12 indicated the graph plotted for throughput value. System throughput is the measure of information moved effectively starting with one spot then onto the next in a given timeframe, and ordinarily estimated in bits every second (bps), as in megabits every second (Mbps) or gigabits every second (Gbps). The comparison values for both the existing and the proposed work come out to be 0.75267952 and 0.806701906666 respectively.

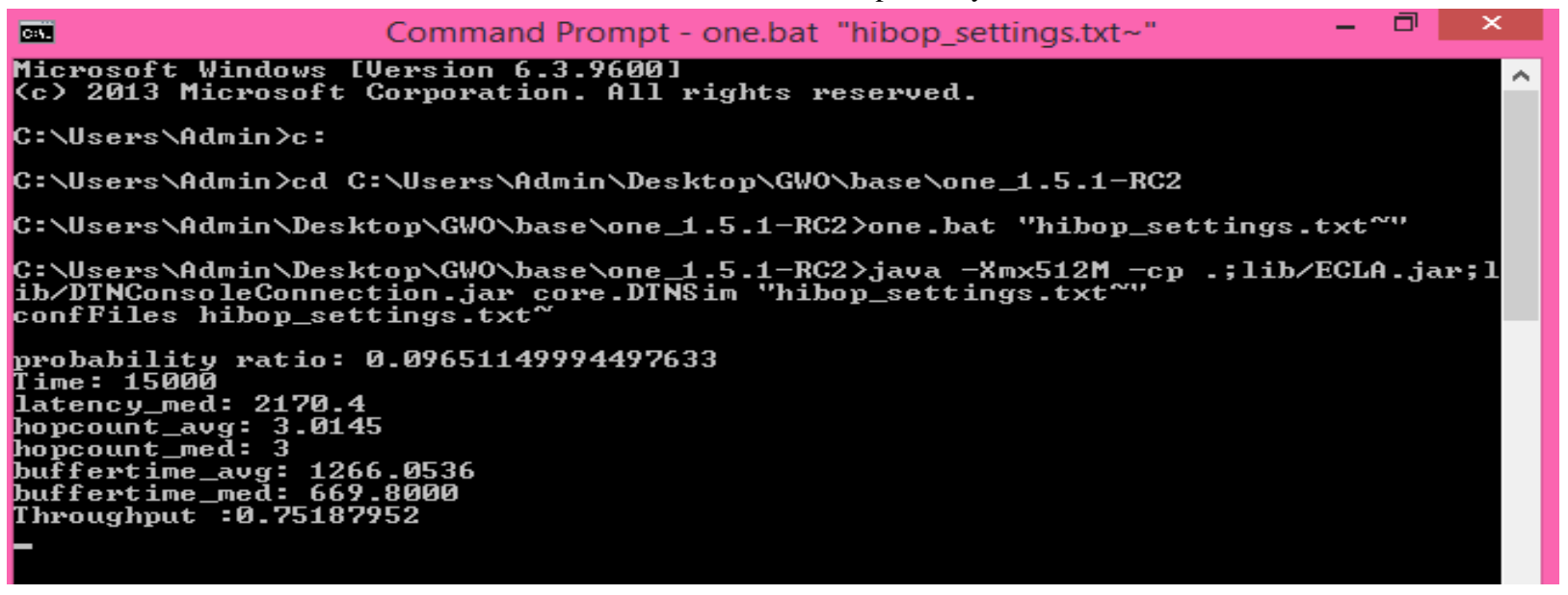

Fig 13: Output Screen of the Existing Work

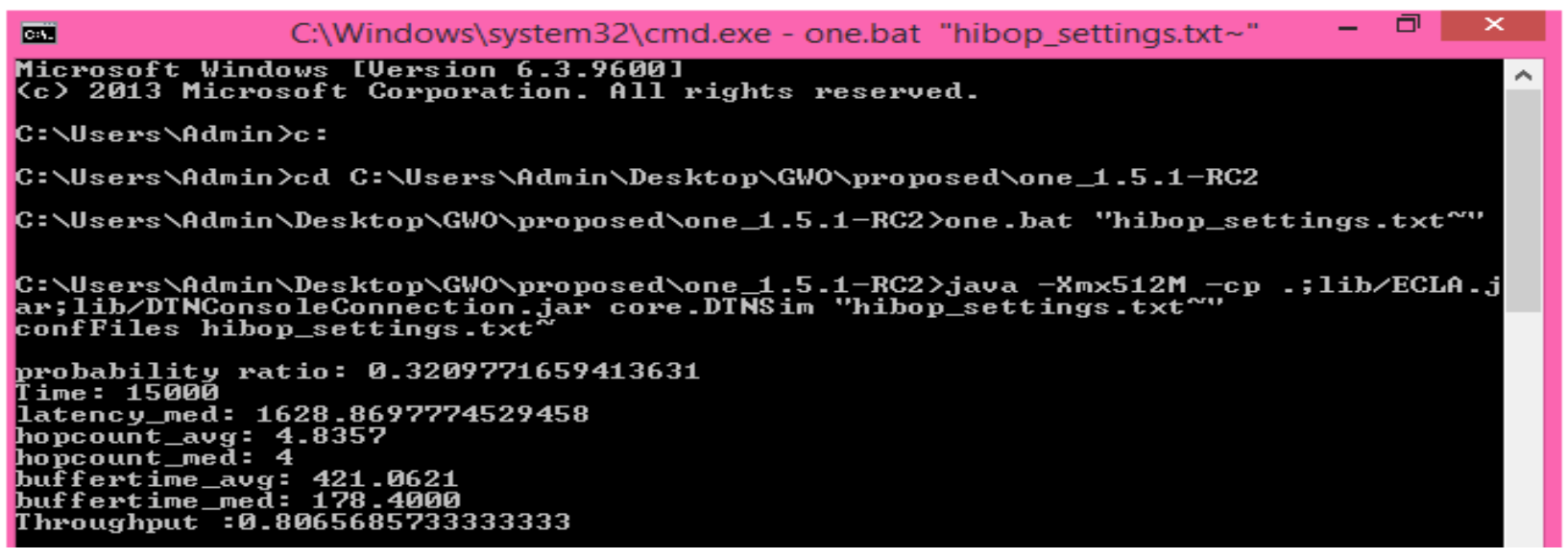

Fig 14: Output Screen of the Proposed Work

Fig 13 and Fig 14 shows the output screens of both the existing-fixed line routing scheme and the proposed GWO work respectively. By comparing these outputs it clearly represents the efficiency and the effectiveness of the GWO applied on the base work.

\section{CONCLUSION}

In this paper, a grey wolf optimization is connected on the fixed-line transportation directing plan dependent on the social qualities. The reenactment results demonstrate that GWO is versatile and accomplishes great system network. The nonstop decrease of search space makes the convergence in GWO quicker and the decision variables are likewise less $(\alpha, \beta$ and $\delta)$. The graph aftereffects of parameters for example throughput, latency, probability of delivery ratio, hop count and buffer time demonstrates that GWO helps in getting compelling and productive routing. The future work is to create social based routing algorithms with respect to real time routers arranged at the vehicles of fixed-line transportation to affirm its sensibility, and to take it to the transportation routing arrangement of non-fixed-line, in which the vehicle drivers of on-fixed-line are generally considered. 


\section{REFERENCES}

[1] Bernsen James and Manivannan D., 2008, "Greedy Routing Protocols for Vehicular Ad Hoc Networks", IEEE.

[2] Bitam Salim, Mellouk Abdelhamid and Zeadally Sherali, 2013, "HyBR: A Hybrid Bio-inspired Bee swarm Routing protocol for safety applications in Vehicular Ad hoc NETworks (VANETs)", Journal of Systems Architecture, Vol.59, Issue:10, science direct.

[3] Bulut Eyuphan and Boleslaw K. Szymanski, 2010, "Friendship Based Routing In Delay Tolerant Mobile Social Networks", IEEE Conference On Global Communication, IEEE.

[4] Chadha Divya, Reena, 2015, "Vehicular Ad hoc Network (VANETs): A Review", International Journal of Innovative Research in Computer and Communication Engineering, Vol.3, Issue:3.

[5] Chebbi, E., Sondi, P., Ramat, E., Rivoirard, L., \& Wahl, M., 2018, "Simulation Of A Clustering Scheme For Vehicular Ad Hoc Networks Using A DEVS-Based Virtual Laboratory Environment", Procedia Computer Science, 130, 344-351.

[6] Chilipirea Cristian, Petre Andreea-Cristina and Dobre Ciprian, 2014, "Social-based routing algorithm for energy preservation in mobile opportunistic networks", International Journal of Embedded Systems.

[7] Chou Li-Der, 2011, "Intersection-Based Routing Protocol For Vanets", Wireless Personal Communications, Vol.60, Issue: 1 .

[8] Cristina Ana Kochem Vendramin, Munaretto Anelise, Regattieri Myriam Delgado, Fonseca Mauro And Carneiro Aline Viana, 2016, "A Social-Aware Routing Protocol For Opportunistic Networks", Expert Systems With Applications.

[9] Gong Haigang, Yu Lingfei, and Zhang Xue, 2014, "Social Contribution-Based Routing Protocol for Vehicular Network with Selfish Nodes", International Journal of Distributed Sensor Networks.

[10] Hom Jacqueline, Good Lauren And Yang Shuhui, 2017, "A Survey Of Social-Based Routing Protocols In Delay Tolerantnetworks", International Conference On Computing, Networking And Communications (ICNC): Workshop, IEEE.

[11] Jerbi Moez, Senouci Sidi-Mohammed, Meraihi Rabah and Ghamri-Doudane Yacine, 2007, "An Improved Vehicular Ad Hoc Routing Protocol for City Environments", IEEE.

[12] Khokhar Rashid Hafeez, Noor Rafidah Md, Ghafoor Kayhan Zrar, Ke Chih-Heng and Ngadi Md Asri, 2011, "Fuzzy-assisted social-based routing for urban vehicular environments", EURASIP Journal on Wireless Communications and Networking, Springer.

[13] Le Tuan And Gerla Mario, 2016, "Social-Distance Based Anycast Routing In Delay Tolerant Networks”, IEEE.

[14] Le Tuan, Kalantarian Haik And Gerla Mario, 2016, “A Novel Social Contact Graph-Based Routing Strategy For Workload And Throughput Fairness In Delay
TolerantNetworks", Wirelesscommunications

And Mobile Computing.

[15] Lenando Halikul and Alrfaay Mohamad, 2018, "EpSoc: Social-Based Epidemic-Based Routing Protocol in Opportunistic Mobile Social Network", Hindawi Mobile Information Systems.

[16] Liu Jianqi, 2016, "A Survey On Position-Based Routing For Vehicular Ad Hoc Networks", Telecommunication Systems.

[17] Liu Li and Jing Yanfang, 2012, "A Survey on Socialbased Routing and Forwarding Protocols in Opportunistic Networks", IEEE.

[18] Luo Yuyi, Zhang Wei, Hu Yangqing, 2010, "A New Cluster Based Routing Protocol for VANET", 2nd International Conference on Networks Security Wireless Communications and Trusted Computing (NSWCTC), Vol. 1, IEEE.

[19] Narang Ghanishtha, Juneja Yogesh , 2015, "Review on classification of different VANET Protocols based on routing information", International Journal of Advanced Research in Computer and Communication Engineering, Vol. 4, Issue 5.

[20] Ramaprabha T., Premalatha V.,2016, ”A Survey On Security Issues And Challenges In VANET", International Journal of Contemporary Research in Computer Science and Technology (IJCRCST), Volume2, Issue 7.

[21] Ren, M., Zhang, J., Khoukhi, L., Labiod, H., \& Vèque, V., 2018, "A Unified Framework Of Clustering Approach In Vehicular Ad Hoc Networks",IEEE Transactions On Intelligent Transportation Systems, 19(5), 1401-1414.

[22] Schoeneich Radosław O. And Surgiewicz Rafał, 2017, "Socialrouting: The Social-Based Routing Algorithm For Delay Tolerant Networks".

[23] Sharma Sheetal, and Sharma Ashish, 2017, “ Improve VANET Routing by Grey Wolf Optimization and Centrality Approach", International Journal Of Research In Electronics And Computer Engineering.

[24] Shi Junling, Wang Xingwei, Huang Min, Li Keqin and Das Sajal K.., 2016, "Social-based routing scheme for fixed-line VANET", science direct.

[25] Soares Vasco N.G.J., Rodrigues Joel J.P.C. and Farahmand Farid, 2011, "GeoSpray: A geographic routing protocol for vehicular delay-tolerant networks", science direct.

[26] Song Youmei, Li Jianbo, Li Chenglong and Wang Fushu, 2016, "Social popularity based routing in delay tolerant networks", international journal on smart sensing and intelligent systems VOL.9, NO.4.

[27] Zhu Ying, Xu Bin, Shi Xinghua, and Wang Yu, 2013, “A Survey of Social-Based Routing in Delay Tolerant Networks: Positive and Negative Social Effects", IEEE Communications Surveys \& Tutorials, Vol. 15, No. 1, IEEE. 